\title{
Ion-Exchange Based Immobilization of Chromogenic Reagents on Microfluidic Paper Analytical Devices
}

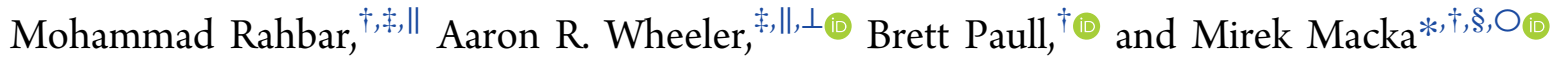 \\ ${ }^{\dagger}$ Australian Centre for Research on Separation Science (ACROSS), School of Natural Sciences, University of Tasmania, Hobart \\ 7001, Australia \\ ${ }^{\ddagger}$ Department of Chemistry, University of Toronto, 80 St. George Street, Toronto, Ontario M5S 3H6, Canada \\ "Donnelly Centre for Cellular and Biomolecular Research, 160 College Street, Toronto, Ontario M5S 3E1, Canada \\ ${ }^{\perp}$ Institute of Biomaterials and Biomedical Engineering, University of Toronto, 164 College Street, Toronto, Ontario M5S 3G9, \\ Canada \\ ${ }^{\S}$ Department of Chemistry and Biochemistry, Mendel University in Brno, Zemedelska 1, CZ-613 00 Brno, Czech Republic \\ ${ }^{\circ}$ Central European Institute of Technology, Brno University of Technology, Purkynova 123, CZ-612 00 Brno, Czech Republic
}

Supporting Information

ABSTRACT: Distance-based detection methods, as used in development of microfluidic paper analytical devices ( $\mu \mathrm{PADs}$ ), rely on the dynamic formation of a colored band along the length of the paper microfluidic channels. The color change is driven by the reaction of chromogenic reagents (typically water-insoluble) that are bound to the paper, thus not subject to being washed away by the sample flow along the detection channel. Here, we introduce the use of an anion-exchange filter paper (as a replacement for standard, unmodified filter paper) for distance-based detection in $\mu \mathrm{PADs}$, in order to immobilize the water-soluble anionic reagents upon the paper detection channels based on ion-exchange interactions of the oppositely charged paper (protonated tertiary amine groups) and the anionic groups of the reagents. The ion-exchange (IE) paper was initially characterized and its properties were compared with standard cellulose paper. The IE paper was shown to be capable of strong retention of anionic reagents exhibiting acidic functional groups (carboxylic, sulfonic), which become deprotonated and negatively charged when in contact with the IE paper. The effect of the ionic strength $\left(10-250 \mathrm{mM} \mathrm{Cl}^{-}\right)$and $\mathrm{pH}(1-13)$ on the immobilization of the investigated reagents were also determined. The IE- $\mu$ PADs were then modified with anionic chromogenic reagents and applied to distance-based determination of total calcium ( $\mathrm{LOD}=0.03 \mathrm{mM}$ ) and total acidity $(\mathrm{LOD}=2.5 \mathrm{mM})$ content in serum and wine samples, respectively. The detailed mechanisms of the developed assays on the IE paper are also discussed. We propose that IE- $\mu$ PADs represent a useful new addition to the distance-based detection toolbox and considerably enhance the applicability of such a detection method.
M icrofluidic paper-based analytical devices ( $\mu$ PADs) have evolved in the past decade to respond to the growing need for low-cost, rapid, portable, and accessible point-of-care diagnostics. The detection step in such devices is typically based on a colorimetric reaction in which a reagent bound to the paper produces a colored product after reacting with target analyte present in the sample. In traditional $\mu$ PADs, quantitative analysis requires digital image colorimetry (DIC), in which color and intensity are related to analyte concentration via a camera and image-processing software. ${ }^{1,2}$ Unfortunately, there are some issues and concerns associated with color development in $\mu \mathrm{PAD}$ analysis, including the "coffee-ring effect" which compromises the homogeneity and intensity of the color pattern, consequently affecting the analytical parameters obtained from DIC. The coffee-ring effect is caused by the fact that there are typically weak attractive forces between the chromogenic reagents (or the colored products of the reaction) and the paper fibers, such that the developed color pattern becomes washed away and/or shifts toward the edges of the detection zone as sample flows through the device. ${ }^{3-5}$ There is great interest in solving this problem by modification of the paper surface by functionalized nanomaterials or oxidizing agents to create attractive forces (e.g., electrostatic interactions) between the reagents (or colored products) and the paper fibers. These efforts have been relatively satisfactory, resulting in more uniform color patterns

Received: March 12, 2019

Accepted: June 5, 2019

Published: June 5, 2019 
and consequently better analytical performance. ${ }^{5-12}$ However, these approaches are not universal and often require relatively tedious preparation steps for each particular assay. Moreover, multiple exposures to different solutions throughout the modification process can deteriorate the mechanical stability and wicking properties of the filter paper (because of the limited wet strength of paper).

A promising alternative to quantitation on $\mu \mathrm{PADs}$ is the use of "distance-based" detection, in which the amount of analyte is converted into a distance signal on a channel, allowing for analysis by eye (with no external instrumentation). ${ }^{13-17}$ However, these methods typically require continuous flow of sample or other solvent through the channel, which exacerbates the challenge of weak interactions between the reagents (or the colored products) and the paper fibers. This challenge is especially problematic at the beginning of the detection channel because of the higher flow rates in this region. ${ }^{18}$ This limits the applicability of the distance-based detection to a limited number of colorimetric reactions, specifically those that rely on water-insoluble chromogenic reagents or colored products that form precipitates or large crystals that can become trapped within the $3 \mathrm{D}$ structure of the cellulose. ${ }^{19-26}$ It has been shown that in order to develop distance-based assays which do not necessarily rely on precipitation reactions but rather involve water-soluble chromogenic reagents, the paper must be extensively modified to retain the reagents and thus to allow formation of a stable distance signal. ${ }^{14}$

In response to the challenges described above, we introduce a new suite of methods for immobilizing chromogenic reagents on distance-based $\mu \mathrm{PADs}$. The new methods rely on anion exchange filter paper (instead of conventional, unmodified filter paper) to allow for convenient electrostatic immobilization of anionic chromogenic reagents (as the most widely used in (bio)chemical assays), such that they will not be washed away by the sample fluid flow. The IE paper used here is modified to present $\mathrm{pH}$-buffered weakly basic constituents, which can cause deprotonation and thus immobilization of acidic reagents such that they become affixed within the paper in the detection channels. This represents a simple, straightforward, and comprehensive approach to immobilization which can be applied to a wide variety of reagents without a specific modification, functionalization, or preparation process for different assays. Herein we describe experiments evaluating the fluidic properties of the IE paper as well as the effect of $\mathrm{pH}$ and ionic strength on displacement of deposited reagents. Most importantly, we evaluate the applicability of the new technique for distance-based $\mu \mathrm{PAD}$ measurements applied to quantifying $\mathrm{Ca}^{2+}$ in serum and total acidity in wine.

\section{EXPERIMENTAL SECTION}

Chemicals and Materials. Unless otherwise specified, reagents were purchased from Sigma-Aldrich (Oakville, ON, Canada), and all aqueous solutions were prepared in deionized water (DI water) with a resistivity $>18 \mathrm{M} \Omega \mathrm{cm}$ obtained from a Millipore (Bedford, MA) Milli-Q water purification system. All chromogenic reagents were in their acid form except 2,6dichlorophenolindophenol (DCPIP) which was in its sodium salt form. Whatman grade 1 and DE81 ion-exchange filter papers (GE Healthcare Australia Pty. Ltd., NSW, Australia) were used for fabrication of the $\mu \mathrm{PADs}$. Transparent laminating film (thickness of $125 \mu \mathrm{m}, \mathrm{GBC}, \mathrm{ON}$, Canada) was used to laminate the $\mu \mathrm{PADs}$.
Measurement of Total Calcium and Acidity. The chromogenic detection reagent solution for the determination of $\mathrm{Ca}^{2+}$ is comprised of arsenazo III $(1 \mathrm{mM})$, TBE $(5 \times)$ buffer, and isopropanol $(10 \% \mathrm{v} / \mathrm{v})$. For the determination of total acidity, bromothymol blue ( $1 \mathrm{mM}$ in $20 \% \mathrm{v} / \mathrm{v}$ ethanol) was used as the colorimetric reagent. In both cases, reagents were deposited upon the detection channels using the pen-plotting approach $^{17,27}$ (plotting speed $=1 \mathrm{~cm} / \mathrm{s}$ ) and were allowed to dry $(2 \mathrm{~min})$ before the final lamination step. Further details regarding instrumentation and fabrication of $\mu \mathrm{PADs}$ can be found in the Supporting Information.

\section{RESULTS AND DISCUSSION}

Characterization of the IE Paper. The DE81 anion exchange filter paper used here comprises a cellulose substrate that is modified with diethylaminoethyl (DEAE) functional groups $\left(\mathrm{p} K_{\mathrm{a}}=9.5\right)^{28}$ which are protonated and therefore positively charged at neutral $\mathrm{pH}$. This type of IE paper has been traditionally used in enzyme assays, DNA polymerase tests, and for separation of anions, nucleotides, and amino acids. Here we introduce the use of DEAE paper as a substrate for fabrication of $\mu \mathrm{PADs}$ to immobilize the anionic colorimetric reagents.

The DEAE paper used here was initially characterized and compared with conventional filter paper for use as a substrate for $\mu$ PADs. First, SEM images revealed no obvious differences between the morphology of the IE paper relative to conventional paper (Figure S1). Then, the new substrates were evaluated for printability, an important property for paper substrates used in the fabrication of $\mu$ PADs. As shown in Figure S2, the IE paper was found to be print compatible and (most importantly) was capable of supporting printed-wax structures that could confine aqueous solutions within them. Finally, flow rate in $\mu$ PADs formed from both types of paper was measured. It was found that the IE paper presented a slightly lower flow rate than conventional filter paper (Figure S3) but one that is well within the range of utility for distancebased detection. This also indicates that the IE paper has similar pore size in relation to the pore size of the standard paper $(11 \mu \mathrm{m})$.

After demonstrating utility for $\mu \mathrm{PAD}$ functionality, attention was turned to evaluating the anion exchange properties of the IE paper. Being aware that the IE paper used in the present work was a weakly basic anion exchanger, an experiment was designed to measure the effective basicity $\left(\mathrm{p} K_{\mathrm{a}}\right)$ of the paper. A series of $\mathrm{pH}$ indicators $\left(\mathrm{p} K_{\mathrm{a}}\right.$ ranging $\left.7.1-11.2\right)$ were selected and their dilute solutions $(0.1 \% \mathrm{w} / \mathrm{v}$ in $20 \% \mathrm{v} / \mathrm{v}$ ethanol) were pipetted onto the paper (Figure S4). Starting from low $\mathrm{pH}$ and moving to high $\mathrm{pH}$, the $\mathrm{p} K_{\mathrm{a}}$ of the first indicator for which the IE paper was not able to convert to its basic form (as realized via a lack of color change) was considered as the effective $\mathrm{p} K_{\mathrm{a}}$ of the paper. As reported by the manufacturer, this value was found to be approximately 9.4. This is important to know, as the $\mathrm{p} K_{\mathrm{a}} \mathrm{s}$ of the paper, the chromogenic reagents, and the analytes must be considered when designing distance-based assays. Further details regarding characterization of the IE paper can be found in the Supporting Information.

Immobilization Tests. Fluorescein was selected as a model anionic analyte to evaluate the capability of the IE paper for electrostatic immobilization. Two spots of fluorescein (0.1 $\mu \mathrm{L}, 0.1 \mathrm{mM}$ in water) were pipetted on the paper channels in IE- $\mu$ PADs, and after drying, water was introduced $(10 \mu \mathrm{L}, 3 \times)$ into the inlets. Importantly, the spots remained intact and no 
elution was observed, indicating a strong interaction between fluorescein and the IE paper surface (Figure 1A). This was
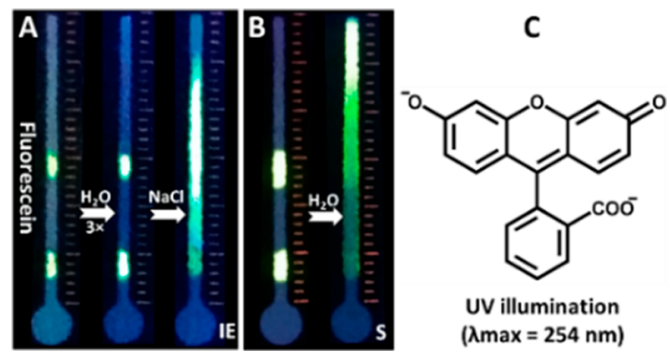

Figure 1. Immobilization test using fluorescein as a model anionic reagent on IE $\mu \mathrm{PAD}$ devices. (A) Representative photographs of fluorescein spots (under UV illumination) on an IE- $\mu$ PAD device after spotting and drying (left), after rinsing in water $(3 \times 10 \mu \mathrm{L})$ (middle), and after rinsing in aqueous $\mathrm{NaCl}(200 \mathrm{mM}, 1 \times, 10 \mu \mathrm{L})$ (right). (B) Representative photographs of fluorescein spots (under UV illumination) on a conventional $\mu \mathrm{PAD}$ device after spotting and drying (left) and after rinsing with water $(1 \times, 10 \mu \mathrm{L})$ (right). (C) Ionized structure of fluorescein comprised of two anionic functional groups anchoring the dye on the positively charged IE paper.

further confirmed by keeping a paper strip impregnated with a spot of fluorescein in water for $2 \mathrm{~h}$ (Figure S5), followed by continuously flushing it with water, where no visible change was observed (Movie S1). However, when chloride $(200 \mathrm{mM}$ $\mathrm{NaCl}$ ) was applied as an eluent to the $\mu$ PADs, the spots were eluted, which confirms the immobilization is based on a simple and reversible ion-exchange mechanism. As a comparison, the same experiment was performed on devices formed from standard filter paper, and the analyte was observed to elute in water even after a single wash (Figure 1B).

The same tests were then applied to another common anionic dye, bromothymol blue (Figure S6 and Movie S2), which showed the same behavior as fluorescein. Identical tests were applied to other reagents (including arsensazo III, calcein, 2,6-dichlorophenolindophenol (DCPIP), and thymol blue) with a range of different functional groups (Figure S7), demonstrating the suitability of the IE paper for immobilization of a wide variety of anionic (acidic) reagents. Note that the amount of deposited reagents per unit area $\left(\mathrm{mol} / \mathrm{mm}^{2}\right)$ in these tests was always negligible compared to the nominal IE capacity reported by the manufacturer $\left(0.017 \mu \mathrm{mol} / \mathrm{mm}^{2}\right)$, which consequently provides complete immobilization of the dye molecules [e.g., $\sim 5 \mathrm{pmol} / \mathrm{mm}^{2}$ for the fluorescein spots in Figure $\left.1\left(\sim 2 \mathrm{~mm}^{2}\right)\right]$. The immobilization tests were also carried out with some cationic dyes (results not shown), such as methylene blue, rhodamine $6 \mathrm{G}$, toluidine blue, neutral red, and nile red, where all were retained on both the standard (as expected) and IE papers. It is worth noting that cellulose naturally presents a strong affinity to cationic dyes due to various interactions, including electrostatic (via the anionic carboxyl and hydroxyl groups of cellulose fibers), hydrogen bonding (through the amino, azo, and amide groups of the typical cationic dyes), van der Waals, and hydrophobic. ${ }^{29}$

Effect of lonic-Strength and pH on Immobilization. IE effects can be changed by sample properties such as ionicstrength and $\mathrm{pH}$; thus, we studied the effects of these variables on the immobilization of indicator dyes. The effect of ionic strength on immobilization was studied by spotting fluorescein $(0.1 \mu \mathrm{L})$ at the beginning of paper channels in IE- $\mu$ PADs and then applying aliquots $(10 \mu \mathrm{L})$ of different concentrations
(10-250 mM) of aqueous chloride ion solution. Chloride was chosen for this demonstration, as it is the primary anion present in many biological samples (e.g., $\sim 100 \mathrm{mM}$ in serum). In these experiments, the distance the dye traveled along the channels was considered as the displacement distance [i.e., dye front $-1.5 \mathrm{~mm}$ (the initial length of the spot)], which was plotted against the concentration of chloride. As is shown in Figure $2 \mathrm{~A}$, there is a strong correlation between chloride
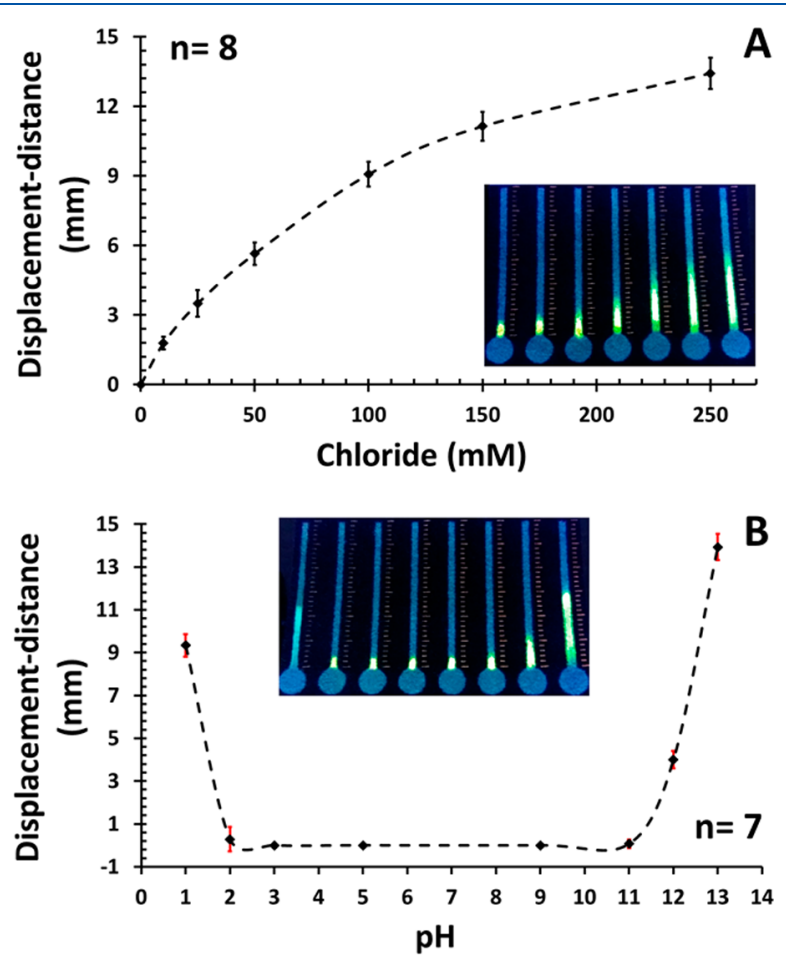

Figure 2. Effect of (A) ionic-strength (chloride ion concentration) and $(\mathrm{B}) \mathrm{pH}(1-13)$ on the immobilization of fluorescein on IE paper. The error bars represent the standard deviations from the average values. Insets are representative photographs (under UV illumination) of $\mu$ PADs used in these experiments (each image corresponds to a data point on the relevant graph).

concentration and the displacement distance. As shown in the inset-images, despite the observed elution, the dye is not completely displaced from its origin, and there is a gradient formed along the channel. This result is useful, as if the reagent is not entirely washed away (with some remaining in the channel), and even a gradient of immobilized reagent might be used (upon some tuning) for development of a distance-based assay.

The effect of $\mathrm{pH}$ on immobilization was investigated for sample $\mathrm{pH}$-effects using methods similar to those described above, i.e., depositing and drying a spot of fluorescein on an IE- $\mu$ PADs, followed by delivery of aliquots $(10 \mu \mathrm{L})$ of different concentrations of $\mathrm{HCl}$ or $\mathrm{NaOH}$ at a range of different $\mathrm{pH}$ values $(1-13)$. The displacement distances for each condition were measured and plotted against the $\mathrm{pH}$ (Figure 2B). As expected, at high $\mathrm{pH}$ (i.e., over 11), the dye was displaced, caused by the titration and deprotonation of the ion-exchange sites (tertiary amine groups) by the sample. For acidic conditions, only $\mathrm{pH} 1$ resulted in displacement, which is likely associated with both the high level of chloride ion $(100 \mathrm{mM})$ present in this $(\mathrm{HCl})$ sample and also the protonation of the carboxyl group of fluorescein that is involved in the ion- 
exchange interaction. Note that the lower intensity of the eluted fluorescein band formed along the channel in this experiment is caused by the diminished fluorescence quantum yield $(\Phi)$ at low $\mathrm{pH}^{30}$ These observations provide a general framework for predicting how the immobilized reagents might be affected in extreme conditions. In addition to the investigated parameters, there might be some other factors playing a role in this context, such as the number, $\mathrm{p} K_{\mathrm{a}}$ and type of anionic functional groups anchoring the dye molecule on the surface of the IE paper. For instance, as shown in the following section, arsenazo III, which contains six anionic functional groups, is retained upon the paper so securely that even samples containing high levels of competing anions do not cause elution.

Distance-Based $\mu$ PAD Assays. As proof of concept, two distance-based assays were developed for implementation on IE- $\mu$ PADs for determination of calcium and total acidity. The former $\left(\mathrm{Ca}^{2+}\right)$ was selected as one of the most prevalent cations in mammals, for example, human serum contains an average $\left[\mathrm{Ca}^{2+}\right]=2.4 \mathrm{mM}$. The determination of the $\mathrm{Ca}^{2+}$ level in serum is important because the deficiency and excessive concentrations are associated with the medical disorders hypocalcemia and hypercalcemia, respectively. ${ }^{31}$ Conventional methods for serum $\mathrm{Ca}^{2+}$ measurement (e.g., photometric methods, atomic absorbance spectroscopy, precipitation techniques, etc.) require multiple preparation steps, large volumes of sample and reagent, and/or bulky instrumentation, which limits their suitability for point-of-care analysis. We propose that IE- $\mu$ PADs with distance detection of $\mathrm{Ca}^{2+}$ offers an instrument-free, low-cost, rapid, and simple alternative that may be appropriate for application at the point-of-care. For distance-based determination of $\mathrm{Ca}^{2+}$, arsensazo III was immobilized upon the IE paper channels. The immobilized dye is purple when not bound to calcium but becomes blue upon exposure to $\mathrm{Ca}^{2+}$-containing sample in the $\mu \mathrm{PADs}$, as depicted in Scheme 1. After introduction of $\mathrm{Ca}^{2+}$ ions, the arsenous and phenolic groups in the dye can complex with $\mathrm{Ca}^{2+}$, while the sulfonate groups (which are not involved in the complex formation), remain free to keep the dye immobilized. As shown in Figure 3A, this system was useful for distancebased determination of $\mathrm{Ca}^{2+}$ in standard solutions of $\mathrm{Ca}^{2+}$

Scheme 1. Mechanism (Top) and Representative Photographs (Bottom) of $\mathrm{Ca}^{2+}$ Assay Performed on IE Paper $^{a}$
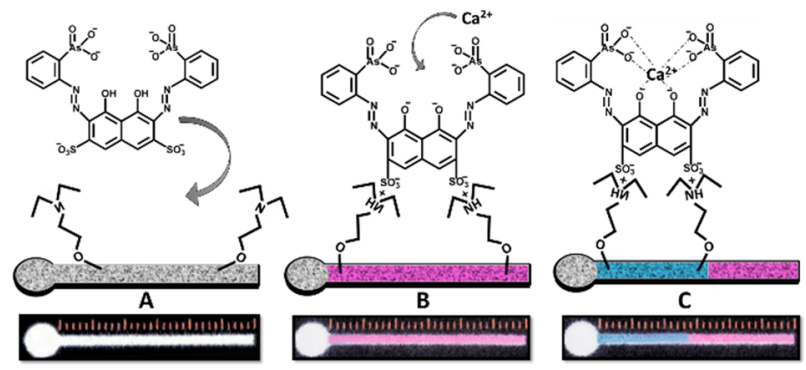

${ }^{a}$ (A) Wax printed IE- $\mu$ PADs. (B) Noncomplexed arsenazo III (pink) is immobilized in the detection channel via its sulfonate groups $\left(\mathrm{p} K_{\mathrm{a}}=\right.$ -2.5 and 0$){ }^{35}$ (Note that the arsenous and phenolic groups might also be involved in the immobilization (due to the low $\mathrm{p} K_{\mathrm{a}}$ values $^{36}$ ), but they are not shown in this manner for simplicity.). (C) $\mathrm{Ca}^{2+}$ containing sample is loaded and wicked from left to right; upon complexation with arsenazo, the dye appears blue.

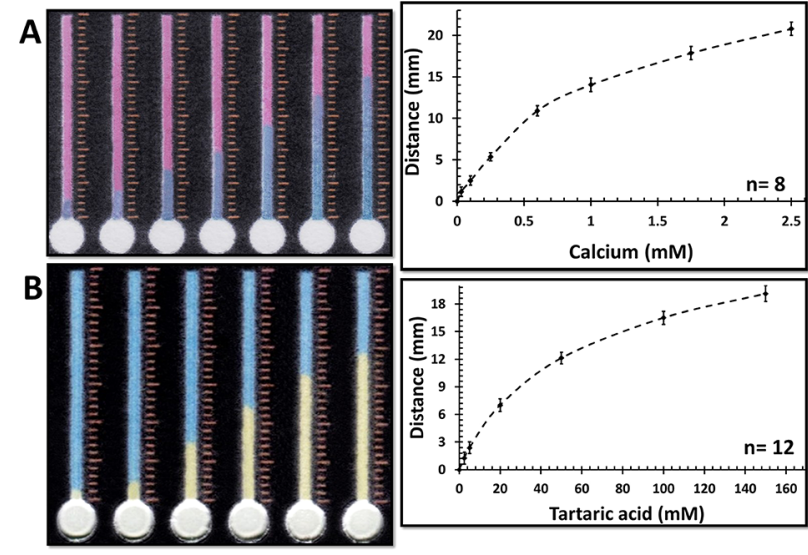

Figure 3. Distance-based measurements on IE- $\mu$ PADs: (left) representative photographs of devices after exposure to standard solutions with increasing concentration of analyte (left-to-right); (right) plots of measured distance as a function of concentration. (A) Aqueous solutions of calcium $(0.03-2.5 \mathrm{mM})$ and (B) aqueous solutions of tartaric acid $(2.5-150 \mathrm{mM})$. The error bars represent the standard deviations from the average values.

(0.03-2.5 mM). The shortest discernible visible colored band formed at the beginning of the detection channel corresponds to $0.03 \mathrm{mM}$, which is comparable to or better than the limits of detection reported previously for $\mathrm{Ca}^{2+}$ in other types of $\mu$ PADs. $^{32,33}$

The second application explored here was motivated by the need for measurements of total or titratable acidity (TA) in the quality assurance of drinks and beverages. TA is the measure of all the various acids present in the sample; however, tartaric acid is the primary acid in grape juices and wines and is often measured as a marker to represent all of the acids that are present. In particular, winemakers strive to maintain a balance between the concentration of acid (normally $\sim 4-10 \mathrm{~g} / \mathrm{L}$ or $\sim 27-67 \mathrm{mM}$ ) and those of other components to reach the desired quality and taste. ${ }^{34}$ TA is typically measured by traditional acid-base titrations relying on $\mathrm{pH}$ indicators or via digital autotitrators with potentiometric detection. Here, we introduce distance-based $\mu \mathrm{PADs}$ as an alternative for rapid, simple, and instrument-free at-site measurement of total acidity. This assay relies on immobilized bromothymol blue (BTB) in IE- $\mu$ PADs. In this case, the IE paper functions not only as an immobilizer but also as a titrant for the distancebased acid-base titration within the paper microfluidic channels. Specifically, as illustrated in Figure S8, BTB is initially anchored on the IE paper via sulfonate and phenolic groups. In this state it is colored blue. After introduction of the acid sample, the phenolic group becomes protonated and is released from the paper; however, the sulfonate group is still bonded to paper allowing formation of a stable yellow-colored band on the paper. In tartaric acid experiments, it was found to be beneficial to cut out the IE-paper in the sample inlets in the $\mu$ PADs. This has the effect of removing the extra IE groups that react with (titrate) the target acids (anions) present in the sample; this results in higher sensitivities (Figure S9). As shown in Figure 3B, upon optimization, standard solutions $(2.5-150 \mathrm{mM})$ of tartaric acid were loaded $(10 \mu \mathrm{L})$ into the sample zone of the $\mu \mathrm{PADs}$ for distance-based signals. The shortest discernible visible colored band formed at the beginning of the detection channel corresponds to $2.5 \mathrm{mM}$, which is considerably better than the previous report on acid 
content measurement via $\mu$ PADs. ${ }^{35}$ Note that improved sensitivity for the IE- $\mu$ PAD should be achievable by preacidifying the immobilized BTB reagent, which would result in lowering the basicity of the IE paper, resulting in longer distances.

After developing IE- $\mu \mathrm{PAD}$ assays for calcium and acid, the tests were applied to determine the total calcium and acid in serum and wine samples, respectively. Specifically, calcium was measured in sheep serum (Quad Five, Ryegate, Mont.) known to contain $2.5 \mathrm{mM} \mathrm{Ca}^{2+}$ (according to the vendor, Figure S10). The serum sample (diluted by water, $10 \times$ ) was applied (10 $\mu \mathrm{L})$ on the distance-based $\mu \mathrm{PADs}$, and the concentration of $\mathrm{Ca}^{2+}$ was found to be $0.31 \pm 0.08 \mathrm{mM}(n=4)$. The total acid assay was tested with white wine samples (Silver Fox Vineyards, Mariposa County, California), in which $10 \mu \mathrm{L}$ of the neat sample was loaded into the inlet of $\mu \mathrm{PADs}$, and the total acid was determined to be $52.3 \pm 1.36 \mathrm{mM}(n=4)$. These measurements were consistent (within $10 \%$ error) with orthogonal measurements of the same samples using a potentiometric autotitrator (Metrohm, Titrando 905), with the total acid determined to be $57.6 \mathrm{mM}(n=4)$.

\section{CONCLUSIONS}

Herein we report the suitability of using ion-exchange filter paper for immobilizing anionic chromogenic reagents through ion-exchange interactions. The immobilization was demonstrated with various reagents comprising different ionizable (acidic) functional groups. The IE paper shows a similar morphology to standard cellulose paper and is printable using standard techniques used to fabricate $\mu$ PADs. The effect of $\mathrm{pH}$ and ionic strength on the displacement of the immobilized reagents were investigated, providing an understanding of the nature of the IE paper used for distance-based assays based on IE immobilization of chromogenic reagents. The immobilization concept was demonstrated by development of distancebased IE- $\mu$ PADs for instrument-free determination of total calcium (cation) and acid (anion) with reasonable analytical performance. This new immobilization concept extends the applicability of distance-based detection to a wider range of chemistries and assays by eliminating the necessity for waterinsoluble chromogenic reagents or precipitated products. In addition, distance-based IE- $\mu$ PADs have the potential to be useful for more complex assays. For instance, in future assays, the sample zone might also function as a sample prep zone, separating (filtering out) anionic interferences, resulting in higher sensitivities.

\section{ASSOCIATED CONTENT}

\section{S Supporting Information}

The Supporting Information is available free of charge on the ACS Publications website at DOI: 10.1021/acs.analchem.9b01288.

Instrumentation, design and fabrication of $\mu \mathrm{PADs}$, and SEM images of filter papers (Figure S1); printability of IE paper (Figure S2); comparison of the flow rate between IE and standard paper (Figure S3); determination of the $\mathrm{pK}$ a of the IE paper (Figure S4); immobilization test: fluorescein (Figure S5); immobilization test: BTB (Figure S6); immobilization test: other reagents (Figure S7); mechanism of total acid assay (Figure S8); effect of sample zone removal on distance signal in total acid assay (Figure S9); and certificate analysis of the sheep serum sample (Figure S10) (PDF) Immobilization test: flushing fluorescein spot (MPG) Immobilization test: flushing BTB spot (MPG)

\section{AUTHOR INFORMATION}

\section{Corresponding Author}

*E-mail: mirek.macka@utas.edu.au. Phone: +61 362266670. Fax: +61362262858.

ORCID

Aaron R. Wheeler: 0000-0001-5230-7475

Brett Paull: 0000-0001-6373-6582

Mirek Macka: 0000-0002-6792-2574

\section{Author Contributions}

The manuscript was written through contributions of all authors.

Notes

The authors declare no competing financial interest.

\section{ACKNOWLEDGMENTS}

We thank the National Sciences and Engineering Council (NSERC) of Canada for support. M.M. gratefully acknowledges the Australian Research Council Future Fellowship (Grant FT120100559). This work was supported by the ESF under Project CZ.02.2.69/0.0/0.0/18_050/0008462.

\section{REFERENCES}

(1) Yang, Y.; Noviana, E.; Nguyen, M. P.; Geiss, B. J.; Dandy, D. S.; Henry, C. S. Anal. Chem. 2017, 89, 71-91.

(2) Yamada, K.; Shibata, H.; Suzuki, K.; Citterio, D. Lab Chip 2017, 17, 1206-1249.

(3) de Freitas, S. V.; de Souza, F. R.; Rodrigues Neto, J. C.; Vasconcelos, G. A.; Abdelnur, P. V.; Vaz, B. G.; Henry, C. S.; Coltro, W. K. Anal. Chem. 2018, 90 (20), 11949-11954.

(4) Morbioli, G. G.; Mazzu-Nascimento, T.; Stockton, A. M.; Carrilho, E. Anal. Chim. Acta 2017, 970, 1-22.

(5) McCann, L.; Benavidez, T. E.; Holtsclaw, S.; Garcia, C. D. Analyst 2017, 142, 3899-3905.

(6) De Tarso Garcia, P.; Garcia Cardoso, T. M.; Garcia, C. D.; Carrilho, E.; Tomazelli Coltro, W. K. RSC Adv. 2014, 4, 3763737644 .

(7) Evans, E.; Moreira Gabriel, E. F.; Benavidez, T. E.; Tomazelli Coltro, W. K.; Garcia, C. D. Analyst 2014, 139, 5560-5567.

(8) Kumar, A.; Hens, A.; Arun, R. K.; Chatterjee, M.; Mahato, K.; Layek, K.; Chanda, N. Analyst 2015, 140, 1817-1821.

(9) Pourreza, N.; Golmohammadi, H. Talanta 2015, 131, 136-141.

(10) Figueredo, F.; Garcia, P. T.; Cortón, E.; Coltro, W. K. T. ACS Appl. Mater. Interfaces 2016, 8, 11-15.

(11) Zhu, X.; Xiong, S.; Zhang, J.; Zhang, X.; Tong, X.; Kong, S. Sens. Actuators, B 2018, 255, 598-604.

(12) Devadhasan, J. P.; Kim, J. Sens. Actuators, B 2018, 273, 18-24.

(13) Cate, D. M.; Dungchai, W.; Cunningham, J. C.; Volckens, J.; Henry, C. S. Lab Chip 2013, 13, 2397-2404.

(14) Yamada, K.; Henares, T. G.; Suzuki, K.; Citterio, D. ACS Appl. Mater. Interfaces 2015, 7, 24864-24875.

(15) Tian, T.; Li, J.; Song, Y.; Zhou, L.; Zhu, Z.; Yang, C. J. Lab Chip 2016, 16, 1139-1151.

(16) Rahbar, M.; Nesterenko, P. N.; Paull, B.; Macka, M. Anal. Chem. 2017, 89, 11918-11923.

(17) Rahbar, M.; Nesterenko, P. N.; Paull, B.; Macka, M. Anal. Chim. Acta 2019, 1047, 115-123.

(18) Channon, R. B.; Nguyen, M.; Scorzelli, A.; Henry, E.; Volckens, J.; Dandy, D.; Henry, C. Lab Chip 2018, 18, 793-802.

(19) Cate, D. M.; Noblitt, S. D.; Volckens, J.; Henry, C. S. Lab Chip 2015, 15, 2808-2818. 
(20) Wei, X.; Tian, T.; Jia, S.; Zhu, Z.; Ma, Y.; Sun, J.; Lin, Z.; Yang, C. J. Anal. Chem. 2015, 87, 4275-4282.

(21) Wei, X.; Tian, T.; Jia, S.; Zhu, Z.; Ma, Y.; Sun, J.; Lin, Z.; Yang, C. J. Anal. Chem. 2016, 88, 2345-2352.

(22) Zhang, Y.; Gao, D.; Fan, J.; Nie, J.; Le, S.; Zhu, W.; Yang, J.; Li, J. Biosens. Bioelectron. 2016, 78, 538-546.

(23) Tian, T.; An, Y.; Wu, Y.; Song, Y.; Zhu, Z.; Yang, C. ACS Appl. Mater. Interfaces 2017, 9, 30480-30487.

(24) Quinn, C. W.; Cate, D. M.; Miller-Lionberg, D. D.; Reilly, T., III; Volckens, J.; Henry, C. S. Environ. Sci. Technol. 2018, 52, 35673573.

(25) Chen, Y.; Chu, W.; Liu, W.; Guo, X. Sens. Actuators, B 2018, 260, 452-459.

(26) Rahbar, M.; Paull, B.; Macka, M. Anal. Chim. Acta 2019, 1063, $1-8$

(27) Nuchtavorn, N.; Macka, M. Anal. Chim. Acta 2016, 919, 7077.

(28) Hettiarachchy, N. S.; Ziegler, G. R. Protein Functionality in Food Systems; CRC Press, 1994.

(29) Roberts, J. C. Paper Chemistry; Blackie: Glasgow, Scotland, 1991.

(30) Martin, M. M.; Lindqvist, L. J. Lumin. 1975, 10, 381-390.

(31) Rifai, N.; Horvath, A. R.; Wittwer, C. T. Tietz Textbook of Clinical Chemistry and Molecular Diagnostics, 6th ed.; Elsevier, 2018.

(32) Ostad, M. A.; Hajinia, A.; Heidari, T. Microchem. J. 2017, 133, 545-550.

(33) Karita, S.; Kaneta, T. Anal. Chim. Acta 2016, 924, 60-67.

(34) Mato, I.; Suárez-Luque, S.; Huidobro, J. F. Food Res. Int. 2005, 38, 1175-1188.

(35) Karita, S.; Kaneta, T. Anal. Chem. 2014, 86, 12108-12114.

(36) Rowatt, E.; Williams, R. J. P. Biochem. J. 1989, 259, 295-298. 